\title{
Splenic Artery Aneurysm: A Retrospective Study of Management in Alexandria University
}

\author{
Ahmed Osmane MD, Sameh Mostafa MD, Naguib ElAskari MD.
}

Department of Vascular Surgery, Alexandria University, Alexandria, Egypt.

Background: Splenic artery aneurysms (SAAs) are rare but are increasingly being diagnosed as incidental findings. They account for around 60\% of visceral artery aneurysms and are the third most common intra-abdominal aneurysms after aortic and iliac aneurysms. The true incidence of SAA is difficult to determine as the majority of cases are asymptomatic. It is clear, however, that SAAs are being found more frequently and identified earlier due to the availability of advanced imaging techniques. The appropriate treatment for SAAs depends on the location and size of the aneurysm, operative risks, and clinical status. The treatment of symptomatic SAAs should be performed. However, no consensus has been reached regarding intervention in asymptomatic patients.

Aim of work: Was to study the appropriate and different ways of management of splenic artery aneurysms.

Patients and methods: 13 patients in ten years attended the main hospital of Alexandria University with a diagnosis of splenic artery aneurysm. Assessed risk factors included age, gender, hypertension, diabetes, hyperlipidaemia, gallbladder diseases, coronary artery diseases, and tobacco use. Patients were also assessed for whether SAA was an incidental finding, symptomatic, or ruptured at presentation. The role of duplex scanning and/or computed tomography (CT) was successful in distinguishing pseudoaneurysm from true one. Calcification, thrombosis, and postoperative splenic infarction were assessed with the assistance of reconstruction of axial imaging. Surgical treatment depended on the site of the aneurysm. Aneurysms located in the proximal or middle third of the splenic artery were treated with simple excision, with proximal and distal ligation of the artery and with splenic preservation. The spleen was preserved only after observation of good back-flow in the distal portion of the splenic artery, and the colour of the spleen was not changed. For aneurysms located in the distal third, resection with splenectomy was performed.

Results: The mean age at diagnosis was 66 years, and 8 of 13 patients (62\%) were females. Co-morbidities were gallbladder diseases (69\%) and systemic hypertension (54\%), however to a less extent were cigarette smoking (54\%), portal hypertension (46\%) and hyperlipidaemia (46\%). 77\% of the patients were complaining of abdominal symptoms, varying from dyspepsia, abdominal discomfort and attacks of upper abdominal pain. One patient was haemodynamically unstable. Two patients were diagnosed incidentally. The size of the splenic artery aneurysms were between $8 \mathrm{~mm}$ and $120 \mathrm{~mm}$ with mean of $26 \mathrm{~mm}$ in diameter. Most of the aneurysms (85\%) were located in the middle and the distal third of the splenic artery; however the proximal third was affected in only $2(15 \%)$ patients. Calcification of the wall of the aneurysm was present in 10 (83\%) patients. Open surgery was done for all patients, and the lesser sac was opened to approach the splenic artery. Double ligation of the splenic artery and splenectomy was the strategy except in two patients where the aneurysm was in the proximal 1/3 of the splenic artery. In these two patients only double ligation-excision of the aneurysm was done with preservation of the spleen. The spleen colour was not changed and fair backflow from distal part of the splenic artery before ligation was present. Post-operative course was smooth for all patients. Pain was present in all patients and was controllable by analgesia. Patients were discharged after 4-7 days from surgery. 
Conclusion: SAAs are usually silent, but their diagnosis nowadays is increasing due to the presence of good imaging techniques. Risk factors for growth and rupture of SAAs should be identified for prophylactic early intervention. Many forms of management have been reported, however open surgery is still favoured.

\section{Introduction:}

Splenic artery aneurysms (SAAs) are rare but increasingly being diagnosed as incidental findings. ${ }^{1}$ They are the third most common abdominal aneurysm preceded only by aortic and iliac artery aneurysms, and comprise around $60 \%$ of visceral artery aneurysms. ${ }^{2}$

Beaussier $^{3}$ reported the first case of splenic artery aneurysm in 1770 at necropsy. It is clear, however, that SAAs are being found more frequently and identified earlier due to availability of high-resolution imaging techniques. ${ }^{4}$ The true prevalence data are widely varying, from $0.01-10.4 \%$ in a large autopsy series. $^{5}$

SAAs are usually seen in patients 60 years or older, they are four times more common in women but approximately three times more likely to rupture in men. ${ }^{6,7}$

Although the precise aetiology of SAAs remains unknown, it has been associated with hypertension, portal hypertension, liver cirrhosis, pregnancy, vasculitis, arterial fibro-dysplasia, and inflammatory and infectious disorders. ${ }^{8,9}$ Primary degeneration of the media secondary to atherosclerosis is considered to be the underlying cause in around $90 \%$ of SAAs. ${ }^{10}$ True aneurysms of splenic artery are usually smaller than $3 \mathrm{~cm}$; they may be multiple and are most commonly located in the distal portion of the artery. Peripheral calcification and mural thrombus may be present. The causes of splenic artery pseudo-aneurysms include pancreatitis, trauma, post laparotomy, and rarely, peptic ulcer disease. ${ }^{11}$ Pancreatic enzymes are thought to cause necrotizing arteritis with destruction of vessel wall architecture and fragmentation of elastic tissues, leading to aneurysm or pseudo-aneurysm. ${ }^{12,13}$

Clinically, most SAAs are asymptomatic. Recent data suggest rupture rates closer to $2-3 \%$. The frequency of rupture is

increased with liver transplantation, portal hypertension, and pregnancy. ${ }^{14-16}$ Ruptured aneurysm is usually presenting by abdominal pain, haemodynamically instability, and gastrointestinal bleeding. 'Double rupture' phenomenon is spontaneous stabilization and subsequent sudden circulatory collapse, as initial bleeding tamponades into the lesser sac, followed by flooding into the peritoneal cavity. ${ }^{17}$ When symptoms are presented incidentally; as abdominal pain, dyspepsia, haematochesia or melena, haemorrhage into the pancreatic duct, and hematemesis; pseudo-aneurysm is suspected with higher risk of rupture even in small diameters. ${ }^{18,19}$

The appropriate treatment for SAAs depends on the location of the aneurysm, the age of the patient, operative risks, and clinical status. The treatment of symptomatic SAAs should be performed. However, no consensus has been reached regarding intervention in asymptomatic patients. ${ }^{20}$

\section{Aim of the work:}

Was to study the appropriate and different ways of management of splenic artery aneurysms.

\section{Patients and methods:}

13 patients attended the main hospital of Alexandria University between January 2002 and December 2011 presented with a diagnosis of splenic artery aneurysm were identified and reviewed.

Preoperative demographic data and clinical variables were collected. Assessed risk factors included age, gender, hypertension, diabetes, hyperlipidaemia, gallbladder diseases, coronary artery diseases, and tobacco use. Only a single female was of child bearing age with negative pregnancy testing at time of hospitalization.

Patients were also assessed for whether 
SAA was an incidental finding, symptomatic, or ruptured at presentation. Aneurysms were considered incidental findings if the patient was receiving an imaging study for a separate established diagnosis.

Preoperative and postoperative imaging consisted of duplex scanning and computed tomography (CT) or both of them.

The role of imaging was great in distinguishing pseudoaneurysm from true one. Imaging criteria were based on the presence of focal arterial disruption and surrounding inflammation in the setting of an irregular aneurysmal wall. Calcification, thrombosis, and postoperative splenic infarction were assessed with the assistance of reconstruction of axial imaging. Calcification was graded subjectively as (0) for no calcification, (1) for $<40 \%$ circumferential calcification, or (2) for $>40 \%$ circumferential calcification based on established scoring methods. ${ }^{21}$ Also data on the hepato-biliary system as liver fibrosis or cirrhosis, portal hypertension, pancreatitis, and gallstones was collected if present in image findings.

In our study, the appropriate surgical treatment depended on the site of the aneurysm. Aneurysms located in the proximal or middle third of the splenic artery were treated with simple excision, with proximal and distal ligation of the artery and splenic preservation. Spleen was preserved only after observation of good back-flow in the distal portion of the splenic artery, and the colour of the spleen was not changed denoting good short gastric vessels. For aneurysms located in the distal third, resection with splenectomy was performed. All patients were operated under general anaesthesia via laparotomy incision, reaching the splenic artery after opening the lesser sac.

\section{Results:}

Diagnosis and management of SAAs were done for 13 patients during this study interval. The mean age at diagnosis was 66 years, and $8(62 \%)$ patients were females and 5 (38\%) were males Table (1). The most associated co-morbidities were gallbladder diseases $(69 \%)$ and systemic hypertension
(54\%), however to a less extend cigarette smoking (54\%), portal hypertension (46\%) and hyperlipidaemia (46\%) Table (2).

$10(77 \%)$ out of 13 patients were complaining of abdominal symptoms, varying from dyspepsia, abdominal discomfort and attacks of upper abdominal pain. One patient (8\%) was haemodynamically unstable, resuscitated and transported to imaging unit and free intraperitoneal collection was found with suspicion of $29 \mathrm{~mm}$ splenic artery aneurysm rupture, and this diagnosis was confirmed intraoperative on abdominal exploration. The other two patients (15\%); one of them was diagnosed incidentally on doing formal abdominal exploration for road traffic accident (RTA) and excision of the aneurysm with splenectomy was performed after repair of a liver tear, however the other patient was diagnosed by imaging done for right ureteric stone Table (3).

Size of the splenic artery aneurysms was between $8 \mathrm{~mm}$ and $120 \mathrm{~mm}$ with mean of $26 \mathrm{~mm}$ in diameter. Most of the aneurysms $(85 \%)$ were located in the middle and the distal third of the splenic artery; however the proximal third was affected in only 2 (15\%) patients. Concerning the calcification of the wall of the aneurysm and according to established scoring method, ${ }^{21} 2(17 \%)$ with no calcification, $6(50 \%)$ with less than $40 \%$ circumferential calcification and $4(33 \%)$ with more than $40 \%$ circumferential calcification Table (4).

Open surgery was done for all patients via abdominal laparotomy, and lesser sac was opened to approach the splenic artery. The accidentally discovered two patients was operated as the size of the aneurysm was 24 and $27 \mathrm{~mm}$ respectively. Double ligation of the splenic artery and splenectomy was the strategy except in two patients where the aneurysm was in the proximal $1 / 3$ of the splenic artery. These two patients onlydouble ligation-excision of the aneurysm was done with preservation of the spleen. The spleen colour was not changed and fair backflow from distal part of the splenic artery before ligation.

Post-operative was smooth for all patients. 
Pain was present in all patients and was controllable by analgesia. Patients were discharged after 4-7 days from surgery. No post-operative fever or wound complication was noticed.

\section{Discussion:}

The pathogenesis of SAAs has not yet cleared. Histological examinations have demonstrated internal and external laminae disruption; fibrodysplasia of the media, subendothelial thickening and glycosaminoglycans deposition. Irrespective of this mechanism, the main issue remains the clinical management of patients and the timing of any intervention. ${ }^{22}$

Lakin et al, ${ }^{23}$ showed more prevalence in females $(69.5 \%)$ with mean age 61 years, and this was nearby our findings as females were presenting $(62 \%)$ with mean age 66years at diagnosis. However, in a large Mayo Clinic study, Abbas et al, ${ }^{7}$ found a higher incidence in females $(80 \%)$ with mean age of presentation of 60.6 years. The commonly listed risk factors include atherosclerosis, gallbladder diseases, pancreatitis and portal hypertension. Splenic artery pseudoaneurysm formation has been associated with a history of pancreatitis, trauma or surgical instrumentation. ${ }^{22,24}$

Arteriography still remains the most valuable investigative modality for diagnosis of SAAs. An additional value is the detection of the amount of calcification. Lakin et al detected the inverse correlation of SAA calcification and initial aneurysm size. The more the calcification, the smaller the aneurysm is at presentation. However, no correlation could be detected between calcification and aneurysm rupture, but a conclusion may be that egg-shell calcification early in aneurysm will prevent further expansion.

The treatment of SAAs is recommended in all symptomatic patients. Asymptomatic patients need treatment are those presented with an expanding aneurysm, women of childbearing age, liver transplantation candidates, and thosewith $>2 \mathrm{~cm}$ in diameter. ${ }^{18,25,26}$ The main complication of SAAs is rupture with reported incidence of $6 \%$ with the highest incidence of rupture in young pregnant women. Open surgical excision of the aneurysm, with or without splenectomy, is the conventional treatment which has well documented efficiency and durability. ${ }^{27,28}$ Reports recommend aneurysmectomy with splenectomy if the aneurysm is located in the distal part of the splenic artery, while spleen preservation could take place in middle and proximal parts. In our study, for fear from splenic infarction, spleen preservation was performed only after confirmation of good backflow from distal portion of splenic artery and be sure of no change of the colour of the spleen. Successful laparoscopic resection of a SAA via the anterior or posterior approach has been documented with good exposure, rapid postoperative recovery and preservation of splenic function.

Endovascular approach via selective catheterization of the splenic artery with placement of covered stent is the most advanced, however coil or gel-foam embolization of the aneurysm is a viable option, with risks of splenic infarction, abscess formation, higher rates of recurrence, and endovascular treatment is generally not recommended for larger aneurysms. ${ }^{29}$

\section{Conclusion:}

SAAs are rare clinical condition which is usually silent, but their diagnosis nowadays is increasing due to the availability of advanced imaging techniques.

The natural history and risk factors for rapid growth of SAAs should be identified to detect patients who in need of early intervention.

Several forms of management have been reported, however conventional open surgery is still favoured.

\section{Reference:}

1- Casadei R, Antonacci N, Calculli L, et al: Thrombosed splenic artery aneurysm simulating a pancreatic body mass: Can two entities be distinguished preoperatively thus avoiding diagnostic and therapeutic mistakes. JOP 2007; 8: 235-259.

2- Tochii M, Ogino H, Sasaki H, et al: Successful surgical treatment for aneurysm of splenic 


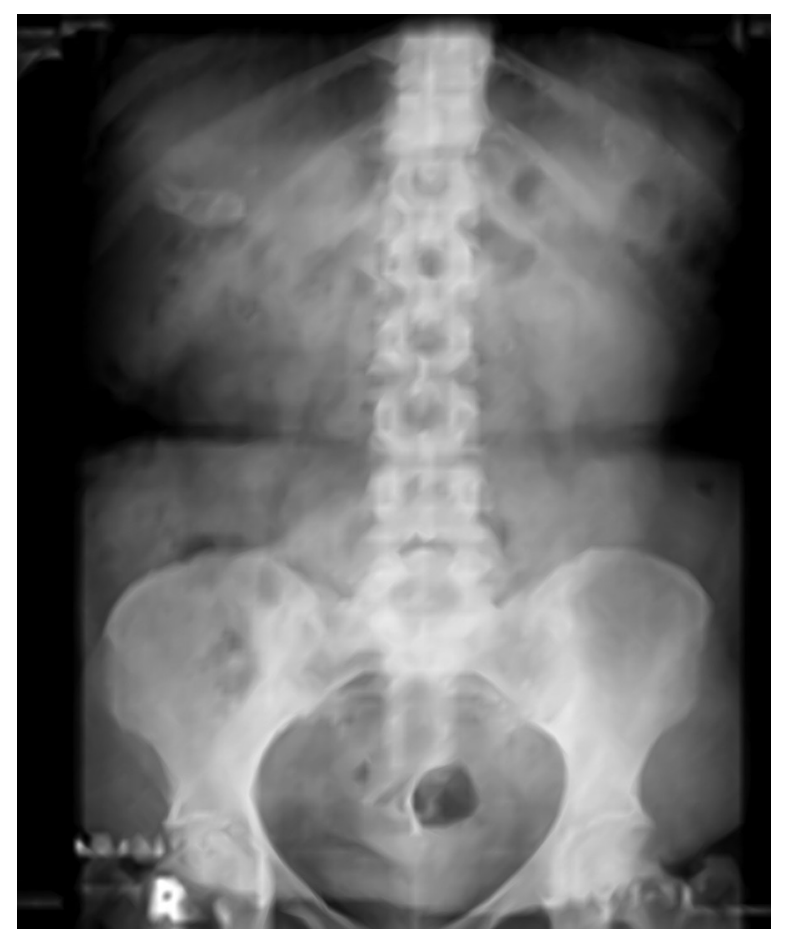

Figure (1): Plain X-ray abdomen and pelvis shows shadow of calcified splenic artery aneurysm together with gallbladder stones.

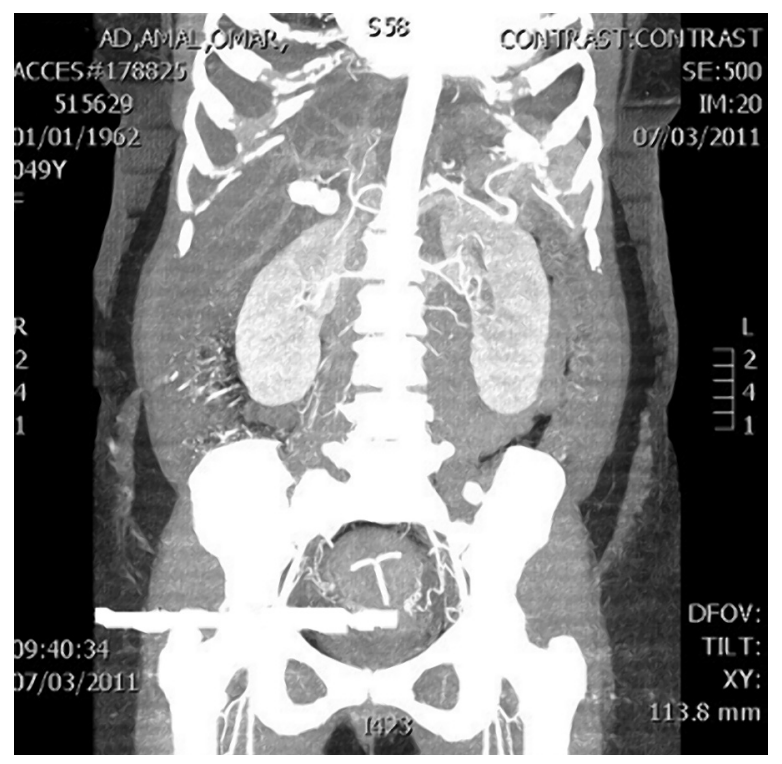

Figure (3): CT with intravenous contrast confirming splenic artery aneurysm.

artery with anomalous origin. Ann Thorac Cardiovasc Surg 2011; 11: 346-349.

3- Beaussier M: Sur un anevrisme de l'artère splénique dont les parois se sont ossifies. $J$ Med Clin Pharm 2009; 32: 157.

4- Kokkalera U, Bhende S, Ghellai A. Laparoscopicmanagement of splenic artery aneurysms. J LaparoendoscAdv Surg Tech 2006; 16: 604-608.

5- Deshpande AA, Kulkarni VM, Rege S, Dalvi

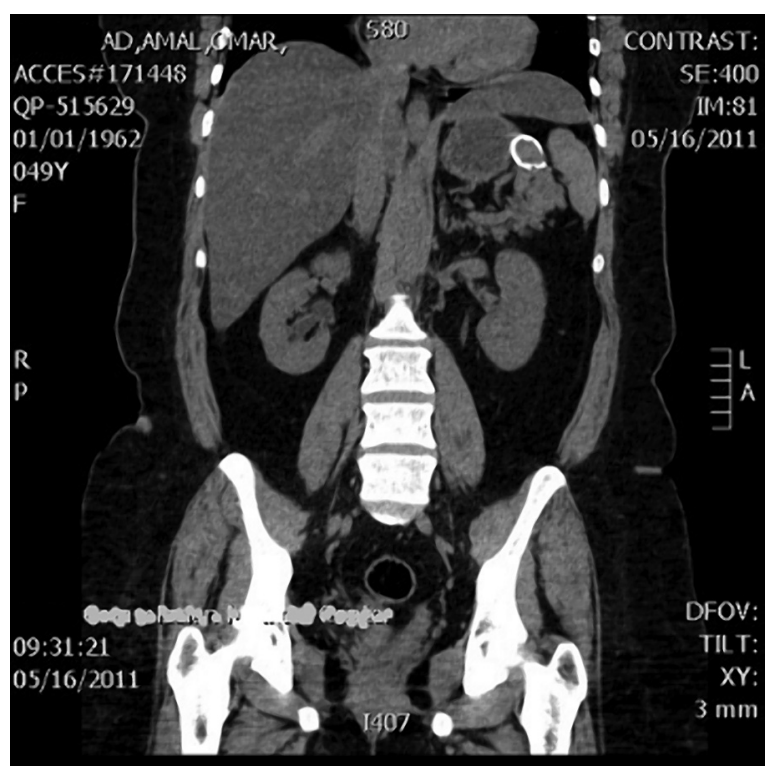

Figure (2): Plain CT abdomen and pelvis showing calcified splenic artery aneurysm.

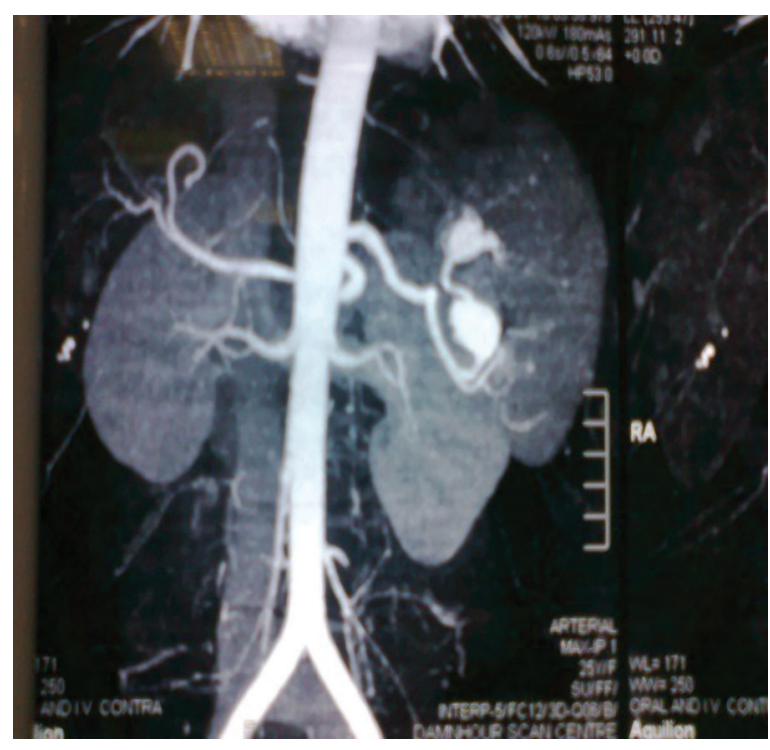

Figure (4): CT with contrast showing multiple splenic artery aneurysms.

AN, Hardikar JV: Ruptured true aneurysm of the splenic artery: An unusual cause of haemoperitoneum. J Postgrad Med 2009; 46: 191-192.

6- Moore SW, Guida PM, Schumacher HW: Splenic artery aneurysm. Bull Soc Int Chir 1970; 29: 210-218.

7- Abbas MA, Stone WM, Fowl RJ, et al: Splenic artery aneurysms: Two decades experience at Mayo clinic. Ann Vasc Surg 


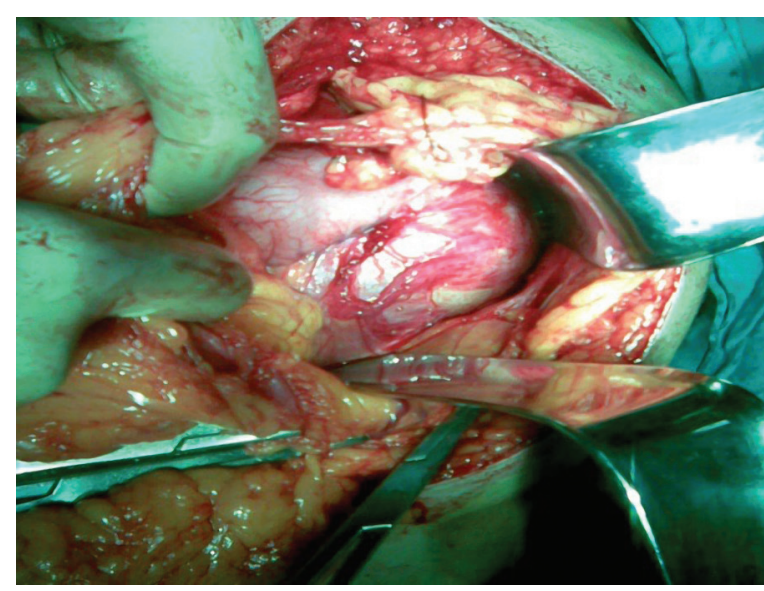

Figure (5): Splenic artery aneurysm exposed after laparotomy.

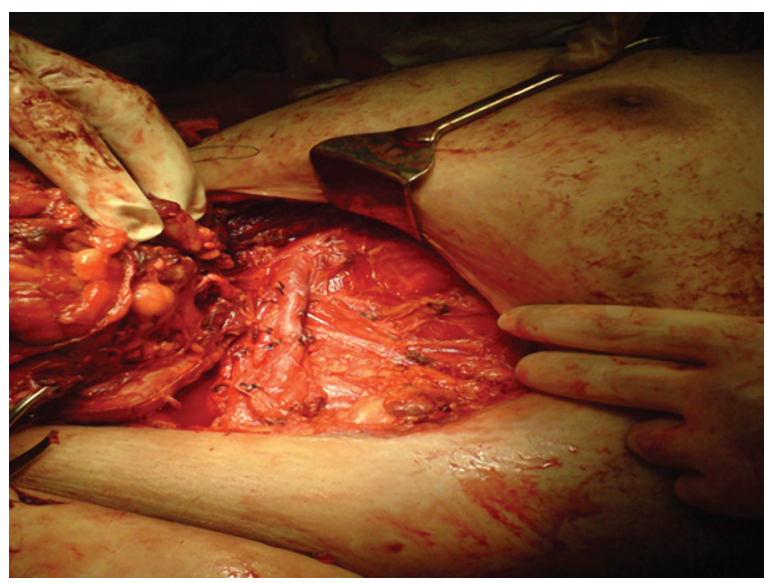

Figure (6): The splenic artery aneurysm dissected and delivered from abdominal cavity.

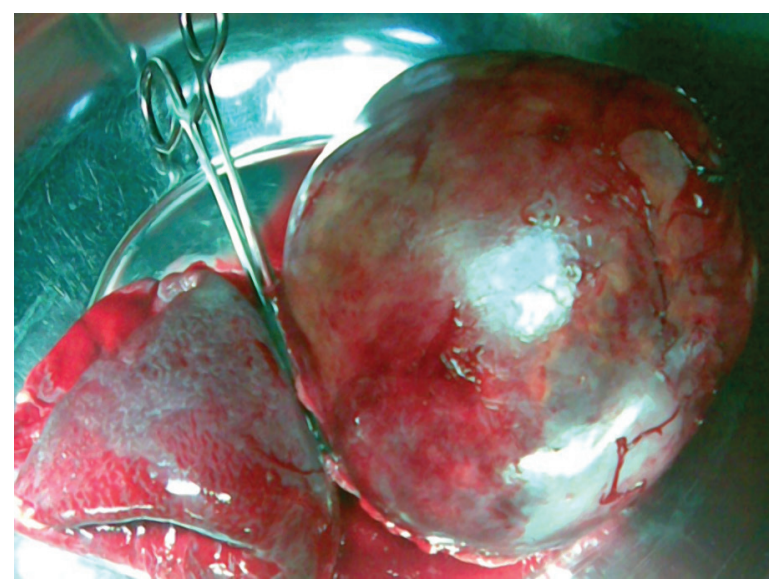

Figure (7): The splenic artery aneurysm and the spleen after total excision.

Table 1: Demographic data on patients with SAAs.

\begin{tabular}{|c|c|c|c|c|}
\hline \multirow[t]{2}{*}{ Variable } & \multicolumn{2}{|c|}{ Total 13 patients } & \multirow{2}{*}{$\begin{array}{c}\text { Test of } \\
\text { significance }\end{array}$} & \multirow{2}{*}{$P$ value } \\
\hline & $\mathrm{N}$ & $\%$ & & \\
\hline $\begin{array}{ll}\text { SEX } & \text { Male } \\
& \text { Female }\end{array}$ & $\begin{array}{l}5 \\
8\end{array}$ & $\begin{array}{l}38 \\
62\end{array}$ & $X^{2}=0.202$ & $\mathrm{FEP}=1.000$ \\
\hline \begin{tabular}{|} 
AGE (year) \\
Min. - Max. \\
Mean +/- SD
\end{tabular} & $\begin{array}{l}48-71 \\
66+/-9.66\end{array}$ & & $\mathrm{~T}=0.110$ & 0.913 \\
\hline
\end{tabular}

SAAs Splenic artery aneurysms

$\mathrm{X}^{2}$ : Value for Chi square, FE: Fisher Exact test, t: Student t-test

2002; 16: 442-449.

8- Lee PC, Rhee RY, Gordon RY, Fung JJ, Webster MW: Management of splenic artery aneurysms: The significance of portal and essential hypertension. J Am Coll Surg 1999; 189: 483-490.

9- Selo-Ojeme DO, Welch CC: Review: Spontaneous rupture of splenic artery aneurysm in pregnancy. Eur JObstet Gynecol
Reprod Biol 2003; 109: 124-127.

10- Dave SP, Reis ED, Hossain A, Taub PJ, Kerstein MD, Hollier LH: Splenic artery aneurysm in the 1990s. Ann Vasc Surg 2000; 14: 223-229.

11- Tessier DJ, Stone WM, Fowl RJ, et al: Clinical features and management of splenic artery pseudoaneurysm: Case series and cumulative review of literature. J Vasc Surg 
Table 2: Risk factors on patients with SAAs.

\begin{tabular}{|l|l|l|}
\hline \multirow{2}{*}{\multicolumn{1}{|c|}{ Variable }} & \multicolumn{2}{c|}{ Total (n=13) } \\
\cline { 2 - 3 } Hypertension & Patients & \% \\
\hline Diabetes & 7 & 54 \\
Hyperlipidaemia & 4 & 31 \\
Coronary artery diseases & 6 & 46 \\
Portal hypertension & 3 & 23 \\
Gallbladder diseases & 6 & 46 \\
Cigarette smoking & 7 & 69 \\
\hline
\end{tabular}

Table 3: Clinical presentations in SAAs.

\begin{tabular}{|l|l|l|}
\hline \multicolumn{1}{|c|}{ Variable } & \multicolumn{1}{c|}{$\mathbf{N}$} & \multicolumn{1}{c|}{$\%$} \\
\hline Abdominal symptoms & 10 & 77 \\
Incidental & 2 & 15 \\
Ruptured & 1 & 8 \\
\hline Total & 13 & 100 \\
\hline
\end{tabular}

Table 4: Imaging data obtained for SAAs.

\begin{tabular}{|l|l|l|l|l|}
\hline \multirow{3}{*}{ Size $(\mathrm{mm})$} & Range & Mean & SD & Total $(\mathrm{n}=13)$ \\
\cline { 2 - 5 } & $8-120$ & 26 & 14 & $13(100 \%)$ \\
\hline \multirow{3}{*}{ Site } & Proximal third & Middle third & Distal third & \multirow{2}{*}{$13(100 \%)$} \\
\cline { 2 - 4 } & $2(15 \%)$ & $4(30 \%)$ & $7(55 \%)$ & \multirow{2}{*}{$12(92 \%)$} \\
\hline \multirow{3}{*}{ Calcification } & 0 & 1 & 2 & \multicolumn{2}{|c}{} \\
\cline { 2 - 4 } & $2(17 \%)$ & $6(50 \%)$ & $43 \%)$ & \\
\hline
\end{tabular}

$\mathrm{SD}=$ Standard deviation;

$0=$ no calcification, $1=<40 \%$ circumferential calcification, $2=>40 \%$ circumferential calcification.

2003; 38: 969-974.

12- Puri S, Nicholson AA, Breen DJ: Percutaneousthrombin injection for the treatment of a post-pancreatitis pseudoaneurysm. Eur Radiol 2003; 13 [suppl 4]: L79-L82.

13- Flati G, Andren-Sandberg A, La Pinta M, Porowska B, Carboni M: Potentially fatal bleeding in acute pancreatitis: Pathophysiology, prevention and treatment. Pancreas 2003; 26: 8-14.

14- Stanley JC, Wakefield TW, Graham LM, Whitehouse WM Jr, Zelenock GB, Lindenauer SM: Clinical importance and management of splanchnic artery aneurysms. J Vasc Surg 1986; 3: 836-840.

15- Trastek VF, Pairolero PC, Bernatz PE: Splenic artery aneurysms. World J Surg
1985; 9: 378-383.

16- Mattar SG, Lumsden AB: The management of splenic artery aneurysms: Experience with 23 cases. Am J Surg 1995; 169: 580-584.

17- Remy D, Linder JL: Splenic aneurysm rupture: Case report and review of the literature. Acta Chir Belg 1993; 93: 54-57.

18- Huang IH, Zuckerman DA, Matthews JB: Occlusion of a giant splenic artery pseudoaneurysm with percutaneous thrombin-collagen injection. $J$ Vasc Surg 2004; 40: 574-577.

19- LiPuma JP, Sachs PB, Sands MJ, Stuhlmiller S, Herbener TE: Angiography/ interventional case of the day: Splenic artery pseudoaneurysm associated with pancreatitis. AJR 1997; 169: 262-263.

20- Busuttil RW, Brin BJ: The diagnosis and 
management of visceral artery aneurysms. Surgery 1999; 88: 619-624.

21- MatsushitaM,NishikimiN, Sakurai T, Nimura Y: Relationship between aortic calcification and atherosclerotic disease in patients with abdominal aortic aneurysm. Int Angiol 2000; 19: 276-279.

22- Tessier DJ, Stone WM, Fowl RJ, Abbas MA, Andrews JC, Bower TC, et al: Clinical features andmanagement of splenic artery pseudoaneurysm: Case series and cumulative review of literature. J Vasc Surg 2003; 38: 969-974.

23- Ryan O, James F, Kashyap VS, et al: The contemporary management of splenic artery aneurysms. J Vasc Surg 2010; 53: 958-965.

24- Sadat U, Dar O, Walsh S, Varty K: Splenic artery aneurysms in pregnancy: A systematic review. Int J Surg 2008; 6: 261-265.
25- Agrawal A, Whitehouse R, Johnson RW, Augustine T: Giant splenic artery aneurysm associated with arteriovenous malformation. J Vasc Surg 2006; 44: 1345-1349.

26- Moyer HR, Hiramoto JS, Wilson MW, et al: Stent graft repair of a splenic artery aneurysm. J Vasc Surg 2005; 41: 893-897.

27- Alomo JM, Gomez MA, Tamayo MJ, et al: Mycotic pseudoaneurysms after liver transplantation. Transplant Proc 2005; 37: 1512-1514.

28- Chiesa R, Astore D, Guzzo G, et al: Visceral artery aneurysms. Ann Vasc Surg 2005; 19: 42-48.

29- Tulysan N, Kashyap VS, Greenberg RK, et al: The endovascular management of visceral artery aneurysms and pseudo aneurysms. $J$ Vasc Surg 2007; 45: 276-283. 